\title{
ESTUDO DA EFICIÊNCIA DE UM REATOR DE DESCARGA POR BARREIRA DIELÉTRICA (DBD), NA PRODUÇÃO DAS ESPÉCIES ATIVAS DO SEGUNDO SISTEMA POSITIVO DO $\mathrm{N}_{2}$
}

\author{
I. A. SOUZA ${ }^{* 1}$, I. O. NASCIMENTO ${ }^{1}$, A. B. NASCIMENTO NETO ${ }^{1}$, L. A. P. NASCIMENTO ${ }^{1}$, J. M. L. SOUZA ${ }^{1}$, T. H. C. \\ $\operatorname{COSTA}^{1}$ e C. ALVES JR ${ }^{2}$ \\ Universidade Federal do Rio Grande do Norte - UFRN \\ Universidade Federal Rural do Semi-Árido - UFERSA \\ ivan_bbismi@hotmail.com*
}

Artigo submetido em abril/2014 e aceito em maio/2015

DOI: $10.15628 /$ holos.2015.2955

\begin{abstract}
RESUMO
Apesar das inúmeras publicações a respeito da versatilidade da técnica de descarga por barreira dielétrica (DBD), principalmente em aplicações emergentes como descontaminação biológica e química, pouco se apresentou sobre a influência dos parâmetros de processos. No presente trabalho é analisada a influência dos parâmetros do processo sobre as espécies ativas do plasma formado quando ar é utilizado como atmosfera. Analisou-se a influência da distância e voltagem aplicada entre eletrodos, bem como a frequência do pulso aplicado. As análises das espécies
\end{abstract}

foram realizadas diagnosticando o plasma por espectroscopia de emissão óptica (EEO). Esses resultados foram correlacionados com dados da potência consumida no processo de produção de plasma, obtidos a partir das figuras de Lissajous. 0 equipamento DBD utilizado possui sua máxima eficiência de produção de plasma atmosférico com a distância entre eletrodos fixada em $0,5 \mathrm{~mm}$ Voltagem de $15 \mathrm{kV}$ e frequência de 500 ou $600 \mathrm{~Hz}$ dependendo da espécie ativa que se queira em maior quantidade $\mathrm{N}_{2}$ $357 \mathrm{~nm}$ ou $\mathrm{N}_{2} 337 \mathrm{~nm}$ respectivamente

PALAVRAS-CHAVE: Plasma DBD, Eficiência da descarga, Frequência, Regime difuso, EEO.

\section{STUDY OF THE EFFICIENCY OF A DBD REACTOR IN THE PRODUCTION OF THE ACTIVE SPECIES OF THE SECOND POSITIVE SYSTEM OF $\mathrm{N}_{2}$}

\begin{abstract}
Despite numerous publications about the versatility of the technique by dielectric barrier discharge (DBD), especially in emerging applications such as biological and chemical decontamination, little is presented on the influence of process parameters. In this paper we analyzed the influence of process parameters on the active species of the plasma formed when air is used as the atmosphere. We analyzed the influence of the distance between electrodes and applied voltage, and the pulse rate applied. The analyzes were performed
\end{abstract}

species diagnosing the plasma by optical emission spectroscopy (OES). These results were correlated with data from the power consumed in the production process of plasma obtained from the Lissajous figures. The equipment used has a maximum DBD production efficiency of atmospheric plasma with the distance between electrodes $0.5 \mathrm{~mm}$ set at $15 \mathrm{kV}$ and voltage frequency of 500 or $600 \mathrm{~Hz}$ depending on the species one wishes active in greater quantity $\mathrm{N}_{2} 357 \mathrm{~nm}$ or $\mathrm{N}_{2}$ $337 \mathrm{~nm}$ respectively.

KEYWORDS: Plasma DBD, discharge efficiency, frequency, Glow Discharge, OES. 


\section{INTRODUÇÃO}

As pesquisas na área de plasma a baixa pressão tem se destacado pela sua versatilidade e importância em aplicações na indústria microeletrônica, automotiva, aeronáutica, entre outras [1]. Entretanto, seu uso em grande escala é limitante devido, principalmente, ao alto custo das instalações em vácuo. Além disso, restringe seu uso em materiais com alta pressão de vapor, como por exemplo, tecidos vivos e líquidos em geral. Diante disso, foram desenvolvidas várias técnicas alternativas de plasma em pressão atmosférica ou subatmosférica, tais como o plasma corona, micro-ondas, micro cátodo oco (MHCD), tocha de plasma e descarga por barreira dielétrica (DBD) [2].

A técnica DBD despontou nos últimos 15 anos, após o grande investimento realizado, principalmente pelo Japão e Coréia do Sul, para pesquisas na fabricação de monitores de TV [3]. Devido à baixa temperatura do gás no processo, houve um crescente uso em aplicações desse plasma na medicina ou em materiais termicamente sensíveis, como polímeros, líquidos, tecidos, esterilização de dispositivos médicos, entre outros [4].

Isso se deve ao fato de que o plasma formado não está termodinamicamente em equilíbrio, ou seja, a energia total não é igualmente distribuída para as espécies do plasma. Embora a temperatura eletrônica seja alta, em torno de $3000 \mathrm{~K}$, a temperatura média do gás é pouco superior à temperatura ambiente, cerca de $500 \mathrm{~K}$ [5]. Dessa forma, maior parte da energia fornecida ao plasma é usada para processos químicos como dissociação e recombinação de moléculas, formando íons, radicais livres, etc [6]. Um plasma com esse caráter tipicamente químico torna-se relevante na modificação de materiais poliméricos e materiais termicamente sensíveis [5,7].

Além disso, outra vantagem desse processo é a pequena distância necessária entre a fonte do plasma e o material a ser tratado, o que facilita e viabiliza seu uso em grandes dimensões em setores industriais mantendo a eficiência e o baixo custo de energia $[3,8]$. Atualmente, os equipamentos que utilizam esta técnica englobam desde canetas para cicatrização $[9,10]$, até equipamentos industriais de grande porte, que podem produzir grande quantidade de ozônio [3,11].

A técnica DBD consiste na aplicação de pulsos de voltagem na faixa de 25 a $30 \mathrm{KV}$ e frequências a partir de $500 \mathrm{~Hz}$ [6], aplicada entre dois eletrodos onde pelo menos um é revestido com material dielétrico. No momento em que a tensão de ruptura é alcançada, várias microdescargas se distribuem na superfície do dielétrico dando origem ao plasma DBD em regime filamentar. Quando essas microdescargas são mais numerosas, homogêneas e distribuídas dão origem ao DBD em regime difuso [12]. É neste último regime que o DBD está sendo apontada como a técnica de plasma mais promissora na área médica [13]. Porém, ainda faz-se necessário o desenvolvimento de pesquisas relacionadas ao domínio e compreensão dos mecanismos químicos, físicos e biológicos da interação entre o plasma não térmico à pressão atmosférica com células, tecidos, órgãos e microorganismos. Como os micro-organismos interagem com as várias espécies ativas, campos elétricos, radiação UV, radicais livres, átomos e moléculas eletronicamente excitados existentes no DBD [4]. 
Identificar os parâmetros adequados a serem utilizados no DBD, bem como as espécies que resultam no plasma é determinante, pois estes influenciam os resultados no tratamento de materiais, como por exemplo, a deposição de filmes [14,15], esterilização de superfícies [16], tratamento superficial de polímeros [16], tratamentos dentários e dermatológicos [17]. Entre outros. Este trabalho teve como objetivo estudar e conhecer como os parâmetros de distância entre eletrodos Frequência do pulso podem melhorar a produção das espécies ativas do plasma em pressão atmosférica. Com base nos resultados foi encontrado dois conjuntos de parâmetros que se destacaram pala quantidade de espécies ativas gerada, estes parâmetros foram, distância entre eletrodos fixada em $0,5 \mathrm{~mm}$ Voltagem de $15 \mathrm{kV}$ e frequência de 500 ou $600 \mathrm{~Hz}$

\section{MATERIAIS E MÉTODOS}

\subsection{Montagem experimental}

O reator DBD (figura 1) consiste em dois eletrodos de cobre, eletrodo superior (ânodo) possui $40 \mathrm{~mm}$ de diâmetro e o eletrodo inferior (cátodo) possui $30 \mathrm{~mm}$ de diâmetro, montado sob um disco de alumina de $56 \mathrm{~mm}$ de diâmetro e $2 \mathrm{~mm}$ de espessura, que atua como dielétrico. Peças de Politetrafluoretileno (PTFE), foram usinadas para alojar os eletrodos e usadas como dois flanges. Os flanges fecham um tubo de quartzo de $110 \mathrm{~mm}$ de comprimento, com diâmetro interno de $75 \mathrm{~mm}$ e parede de $2,5 \mathrm{~mm}$ de espessura, o qual é encaixado nas peças de teflon penetrando $5 \mathrm{~mm}$ em cada peça. O movimento do eletrodo superior (ânodo) é obtido através de uma cremalheira com escala milimétrica que permite variar a distância entre os eletrodos até um valor de $100 \mathrm{~mm}$, durante o funcionamento do reator.

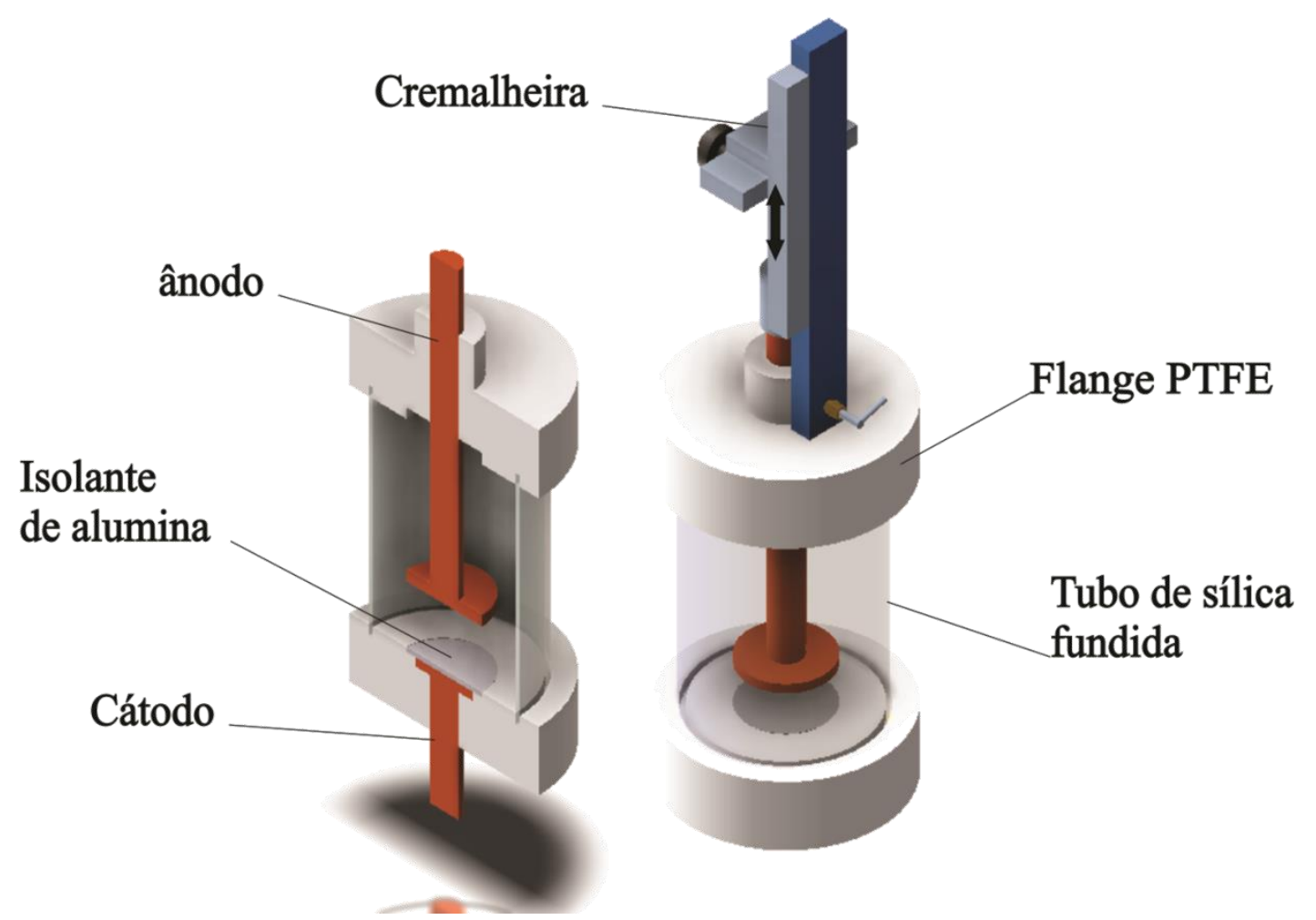

Figura 1: Desenho mostrando os detalhes construtivos do reator DBD utilizado neste trabalho. 
Com o objetivo de variar os parâmetros de voltagem e de frequência, foi utilizada uma fonte de alta voltagem DC pulsada, com largura de pulso de $200 \mu$ s obtendo-se uma melhor eficiência em comparação com as fontes de corrente alternada (AC) [18]. A fonte utilizada permite variar a voltagem aplicada de 0 a $20 \mathrm{KV}$ e frequência de 0 a $1 \mathrm{kHz}$. De acordo com a distância entre os eletrodos, voltagem e frequência utilizada é possível gerar o plasma no interior do reator. Os valores dos parâmetros utilizados neste trabalho foram variados e subdivididos em três etapas a fim de caracterizá-los. Em cada etapa fixava-se dois parâmetros e variava-se o terceiro como mostrado na tabela 1.

Tabela 1: Parâmetros utilizados.

\begin{tabular}{c|c|c|c}
\hline Condições & Distância $\mathbf{( m m )}$ & Frequência (Hz) & Voltagem (kV) \\
\hline D5F6D15 & 0,5 & 600 & 15 \\
D10F6V15 & 1,0 & 600 & 15 \\
D15F6V15 & 1,5 & 600 & 15 \\
D5F5V15 & 0,5 & 500 & 15 \\
D10F5V15 & 1,0 & 500 & 15 \\
D15F5V15 & 1,5 & 500 & 15 \\
D5F4V15 & 0,5 & 400 & 15 \\
D10F4V15 & 1,0 & 400 & 15 \\
D15F4V15 & 1,5 & 400 & 15 \\
D5F2V15 & 0,5 & 200 & 15 \\
D10F2V15 & 1,0 & 200 & 15 \\
D15F2V15 & 1,5 & 200 & 15 \\
\hline
\end{tabular}

\subsection{Analises por EEO}

O estudo foi realizado a partir da Espectroscopia de Emissão Óptica (OES), que é uma ferramenta poderosa quando se trata de avaliar as espécies do plasma $[19,20]$. Os dados ópticos foram obtidos a partir de um espectrômetro modelo USB4000 UV-VIS. A aquisição do espectro foi obtido por uma fibra ótica posicionada entre os dois eletrodos e a uma distância de $1 \mathrm{~mm}$ da borda do ânodo (figura 2), para garantir maior eficiência dos resultados. As respostas espectrais das três etapas de variação dos parâmetros envolvidos nesta pesquisa foram verificadas em tempo real durante o processo de geração do plasma.

\subsection{Medidas elétricas}

As medidas elétricas foram realizadas utilizando-se um osciloscópio modelo MSO-X 2002 2 canais Agilent que permite obter até $1 \mathrm{G}$ amostras/s e resolução de banda de $0.014 \mu \mathrm{s}$, juntamente com uma sonda de alta tensão 1000:1 30KV 50MHz modelo Agilent N2771B. Para calcular a carga transportada em cada ciclo de produção de plasma foi colocado um capacitor de 2,47 nF em série com a saída do reator. Todas as medidas elétricas foram realizadas simultaneamente com as medidas ópticas como ilustrado esquematicamente na figura 3.

O método utilizado para cálculo da energia dissipada e potência consumida no processo foi realizado a partir da Figura de Lissajous [19,20,21]. Para gerar a Figura de Lissajous na tela do osciloscópio, os sinais de voltagem medidos com o auxilio da sonda de alta tensão 1000: 1 e do capacitor ligado em serie com a saída do reator foram colocados em dois canais diferentes osciloscópio. No eixo $X$ foram inseridos os valores da voltagem e no eixo $Y$ os valores da carga, obtidos indiretamente pelas voltagens medidas no capacitor. 


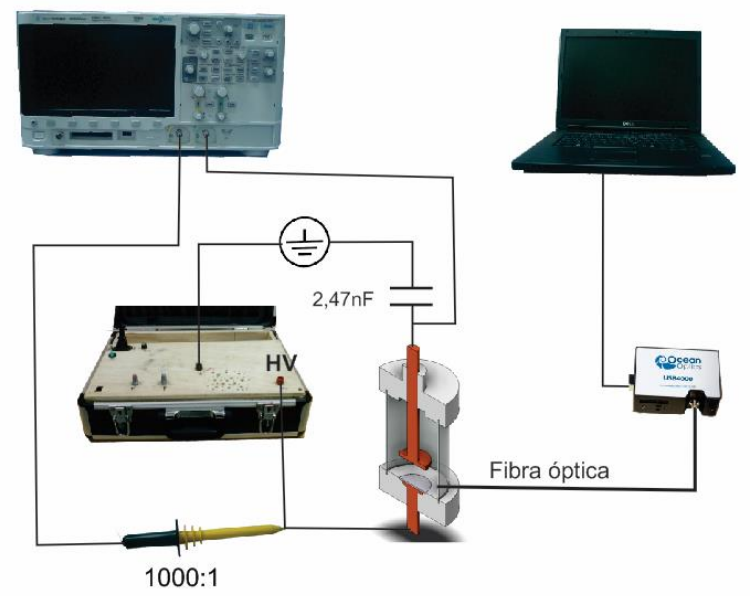

Figura 2: Esquema do arranjo experimental para medidas elétricas e ópticas da descarga DBD utilizada nesse trabalho.

A energia elétrica $E_{e}$ consumida em um ciclo de tensão aplicada entre os dois eletrodos do reator corresponde à área interna da figura de Lissajous (figura 3):

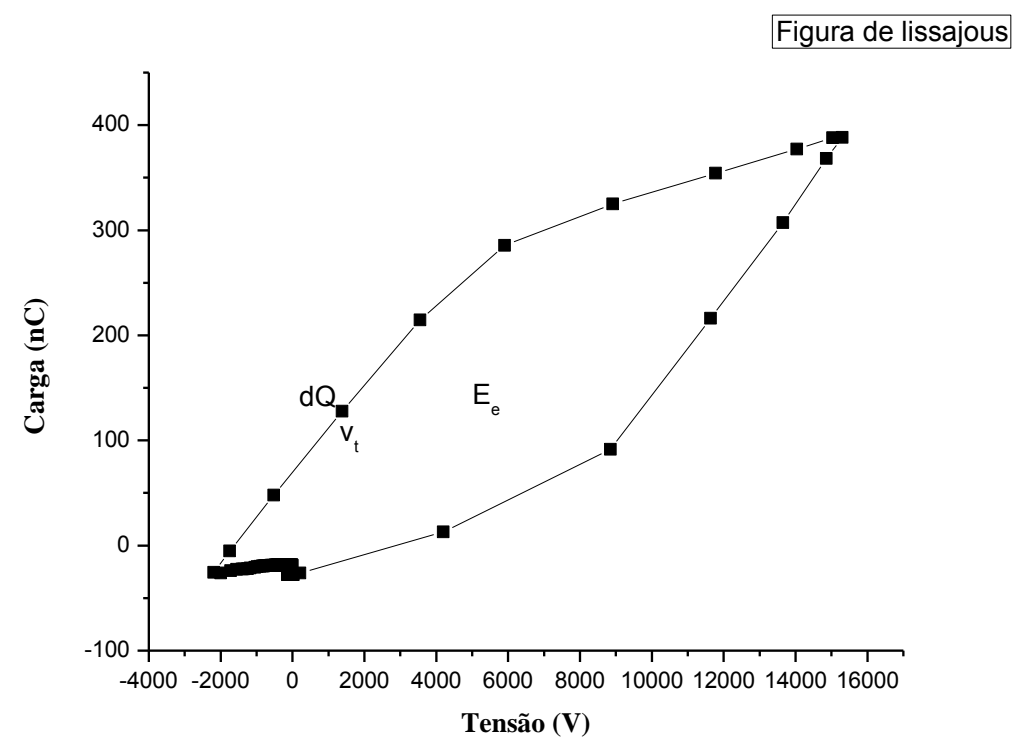

Figura 3: figura de Lissajous.

$$
E_{e}=\int_{0}^{t} V_{t} d Q
$$

Assim a potência $P$ dissipada é obtida multiplicando a energia $E_{e}$ pela frequência do pulso $f_{p}$ ou seja:

$$
P=\int_{0}^{t} V_{t} d Q \cdot f_{p}
$$

Onde $V_{t}$ é a voltagem aplicada nos eletrodos em função do tempo e $d Q$ é um elemento infinitesimal de carga acumulada no capacitor. As medidas ópticas e elétricas foram então obtidas simultaneamente para cada valor de distância e frequência escolhidas. 


\section{RESUTADOS E DISCUSSÃO}

\subsection{Variação da distancia entre eletrodos}

Na figura 4 é mostrado um espectro típico obtido por Espectroscopia de emissão óptica (EEO) no presente trabalho, onde são ilustrados os picos mais intensos. Esse resultado está em acordo com o de vários autores que já diagnosticaram o plasma de ar atmosférico [5,19,23,24]. Esse espectro é composto basicamente de 5 picos correspondentes ao $\mathrm{N}_{2}$ do segundo sistema positivo $\left(C^{3} \prod_{u}-B^{3} \prod_{8}\right){ }^{[1]}$, como também alguns picos do primeiro sistema negativo do $\mathrm{N}_{2}{ }^{+}$ $\left(B^{2} \sum_{u}^{+}-X^{2} \sum_{g}^{+}\right)[5,19]$. Existem relatos que a utilização dessas espécies ativas no plasma, especialmente $\circ \mathrm{N}_{2}$, pode melhorar as propriedades superficiais e o comportamento tribológico dos metais [25], além de agir como agente esterilizador eliminando micro-organismos [26].

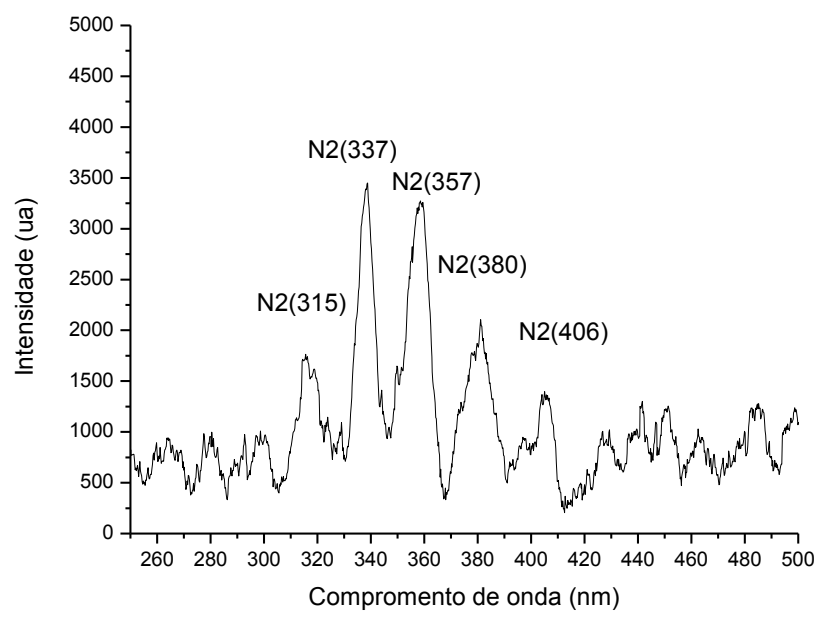

Figura 4: Espectro óptico típico obtido por EEO da descarga DBD aplicada em ar atmosférico.

Das linhas obtidas por EEO, as mais intensas nesta análise foram a do $N_{2}$ (357nm) e $N_{2}$ $(337 \mathrm{~nm})$, seguido de três outros picos, todos na região do UV [28]. Aplicando uma diferença de potencial de $15 \mathrm{kV}$ entre eletrodos, durante 200 us (largura do pulso), numa frequência de 500 $\mathrm{Hz}$, obtém-se os gráficos mostrados nas figuras $5(\mathrm{a})$ e $5(\mathrm{~b})$ para as medidas ópticas e elétricas, respectivamente.
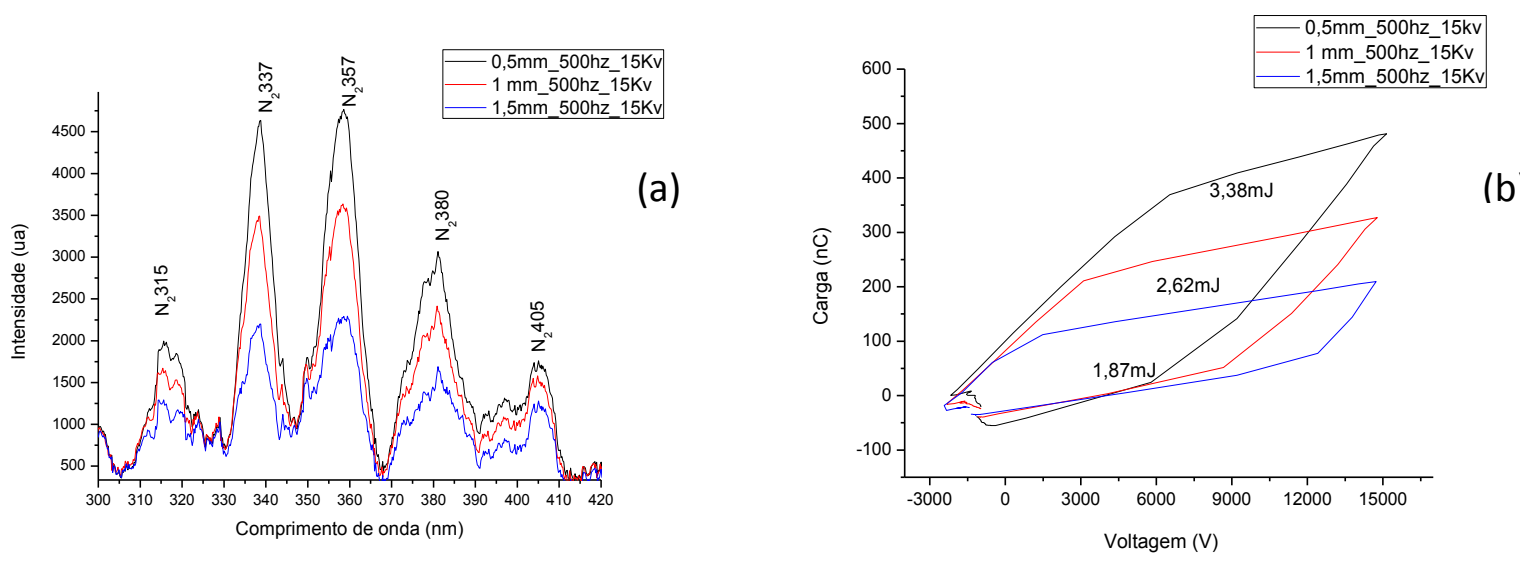

Figura 5: Medidas espectrais e elétricas da descarga entre dois eletrodos, aplicando uma tensão de 15 kV e frequência de $500 \mathrm{~Hz}$. (a) espectro obtido por EEO e (b) figura de Lissajous, destacando a energia por ciclo para cada distância. 
É verificado que para a menor distância entre eletrodos ocorreu maior intensidade espectral. Entretanto, verifica-se que a energia por ciclo nessa condição também é maior, ou seja, embora se gaste mais energia por pulso de tensão, a resposta espectral das espécies ativas é mais intensa. Se por um lado o consumo energético é um fator negativo para as aplicações industriais, a intensidade espectral reflete a densidade de espécies ativas. Assim um parâmetro que forneça uma indicação da densidade de espécies ativas por energia consumida, pode ser de grande importância para avaliação da eficiência de desempenho de um dispositivo DBD. Uma vez que as principais espécies para a descarga em ar atmosférico estão na faixa de $300 \mathrm{~nm}$ a $420 \mathrm{~nm}$, correspondentes às linhas espectrais do $\mathrm{N}_{2}\left(C^{3} \Pi_{u}-B^{3} \Pi_{g}\right)$, a intensidade espectral integrada pode ser fornecido pelo valor da integral:

$$
I_{S}=\int_{300}^{420} I(\lambda) d \lambda
$$

Obtido a partir da área sob a curva da figura 5(a). Assim a razão $I_{S} / E_{E}$ nos fornece e eficiência de um dispositivo DBD [2]. Na tabela 2 são mostrados os valores de $E_{E}, I_{s}$ e $I_{s} / E_{E}$ para todas as três distâncias entre eletrodos estudadas, utilizando distâncias entre pulsos de $5 \mathrm{~ms}, 2,5$ ms, 2 ms e 1,6 ms, correspondentes às frequências de 200, 400, 500 e $600 \mathrm{~Hz}$, respectivamente. Observa-se que para a menor distância entre eletrodos houve maior eficiência do dispositivo $\left(I_{s} / E_{d}\right)$ com exceção apenas para a frequência de $500 \mathrm{~Hz}$, onde a maior eficiência aconteceu para a distância de $1 \mathrm{~mm}$.

Tabela 2: Valores da energia por ciclo (Ee), intensidade espectral integrada(Is) e rendimento do dispositivo DBD (Is/le).

\begin{tabular}{ccccc}
\hline Distância & Frequência & Energia por pulso & $\begin{array}{c}\text { Intensidade espectral } \\
\text { integrada (Is) }\end{array}$ & ${\text { Is/ } \mathbf{E}_{\mathbf{d}}}$ \\
\hline 0,5 & 200 & 2,20 & 28630 & 13013 \\
1 & 200 & 2,80 & 21890 & 7818 \\
1,5 & 200 & 2,86 & 17625 & 6162 \\
0,5 & 400 & 2,30 & 40351 & 17544 \\
1 & 400 & 2,76 & 36300 & 13152 \\
1,5 & 400 & 2,70 & 20364 & 7542 \\
0,5 & 500 & 2,65 & 522389 & 19713 \\
1 & 500 & 1,86 & 37378 & 20096 \\
1,5 & 500 & 1,58 & 25329 & 16031 \\
0,5 & 600 & 2,00 & 59918 & 29959 \\
1 & 600 & 2,40 & 43913 & 18297 \\
1,5 & 600 & 2,78 & 27992 & 10069 \\
\hline
\end{tabular}

Esse aumento da eficiência para distâncias superiores a 0,5 $\mathrm{mm}$ se deve, principalmente, à redução da energia dissipada por pulso. Conforme pode ser verificado na tabela 2, para a frequência de $500 \mathrm{~Hz}$ os valores da energia dissipada possui um comportamento inverso aos demais, ou seja, foram inversamente proporcionais às distâncias entre eletrodos. No entanto, a intensidade integrada possui o mesmo comportamento das demais condições estudadas, ou seja, decai com o aumento da distância entre eletrodos. Observando a figura de lissajous para essa frequência particular (figura 6) pode-se constatar a diferença existente entre a curva obtida quando a distância entre eletrodos era de $0,5 \mathrm{~mm}$ e as duas outras distâncias. Verifica-se que 
para essas últimas, a inclinação dQ/dV é menor, significando uma menor impedância do sistema [2]. Isto significa que essa é a melhor condição de trabalho para a configuração estudada, no que se refere ao casamento de impedância do sistema. Porém, ao analisar o plasma gerado entre os dois eletrodos (figura 7) para essa frequência de $500 \mathrm{~Hz}$, comparativamente com a frequência de $600 \mathrm{~Hz}$, verifica-se que para essa condição o regime do plasma é filamentar, tornando-o pouco interessante para aplicações industriais. Apenas quando os eletrodos estavam a uma distância de $0,5 \mathrm{~mm}$ é que o regime do plasma tornou-se difuso, que é a condição de interesse. Apesar de que nessas condições a energia dissipada foi máxima, isso é justificada pela maior distribuição de espécies no espaço entre eletrodos.

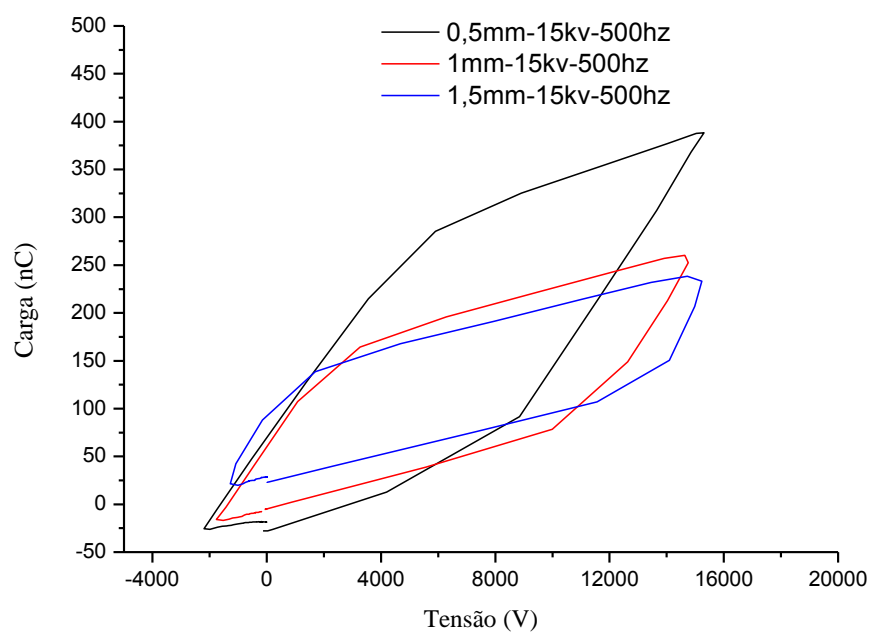

Figura 6: Curva de carregamento do dispositivo DBD pela aplicação de um pulso de voltagem de 15 kV.

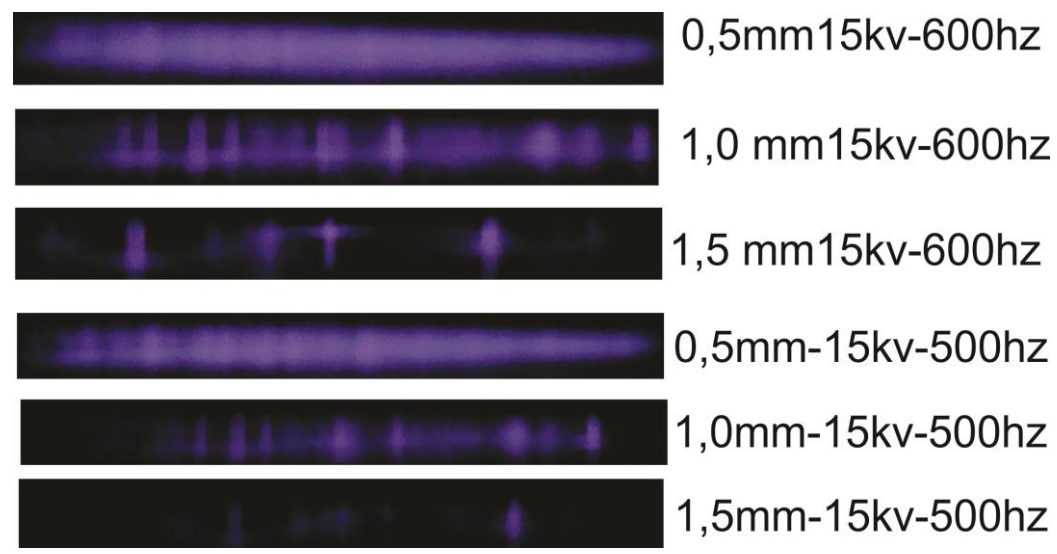

Figura 7: Regimes de operação do DBD atmosférico para as frequências $600 \mathrm{~Hz}$ e $500 \mathrm{~Hz}$.

\section{CONCLUSÃO}

Um equipamento de descarga por barreira dielétrica foi construído e caracterizado fixando a voltagem em $15 \mathrm{kV}$ e variando-se a frequência e distância entre eletrodos. Os resultados das características elétricas e espectrais foram explorados com o intuito de determinar as melhores condições de trabalho do dispositivo. Sugere-se como indicador da eficiência do equipamento a razão entre a intensidade espectral integrada e a energia dissipada por pulso. A intensidade espectral é integrada dentro de uma faixa de comprimento de onda de interesse para a aplicação. Dos resultados encontrados pode-se concluir que: 
As principais linhas espectrais observadas na descarga por barreira dielétrica estudada foi referente ao $\mathrm{N}_{2}$ do segundo sistema positivo do $\mathrm{N}_{2}\left(C^{3} \Pi_{4}-B^{3} \Pi_{g}\right)$ como pode se visto na figura 4 . A tabela 2 mostra de forma clara que maiores intensidades espectrais aconteceram para menores distâncias entre eletrodos e um modo geral a energia por pulso é menor para menores distâncias entre eletrodos. Exceção à regra aconteceu para a frequência de $500 \mathrm{~Hz}$, onde houve uma inversão no comportamento. Esta inversão é justificada pelo surgimento do regime de plasma difuso, que aumenta a densidade espectral entre eletrodos.

A eficiência espectral $\left(I_{s} / E_{e}\right)$ foi maior para a frequência de $500 \mathrm{~Hz}$ e distância de $1 \mathrm{~mm}$. Nessa condição se observou pela figura de Lissajous uma baixa impedância do sistema. Plasma em regime difuso ocorreu apenas para distância entre eletrodos de $0,5 \mathrm{~mm}$ e frequências de 500 $\mathrm{Hz}$ e $600 \mathrm{~Hz}$.

\section{REFERÊNCIAS}

[1] ALVES JR, C. et al. Nitriding of titanium disks and industrial dental implants using hollow cathode discharge. Surface and Coatings Technology, v. 194, n. 2-3, p. 196-202, 5/1/ 2005.

[2] SOUZA, I. A. DESCARGA EM BARREIRA DIELÉTRICA: CONSTRUÇÃO DE UM REATOR DBD E CARACTERIZAÇÃO MEDIANTE ANÁLISES ÓPTICAS E ELÉTRICAS DO PLASMA PRODUZIDO. 2013. 79 (Mestrado). Programa de Pós-Graduação em Engenharia Mecânica, Universidade Federal do Rio Grande do Norte

[3] KOGELSCHATZ, U. Collective phenomena in volume and surface barrier discharges. Journal of Physics: Conference Series, v. 257, n. 1, p. 012015, 2010

[4] FRIDMAN, G. et al. Applied Plasma Medicine. Plasma Processes and Polymers, v. 5, n. 6, p. 503-533, 2008.

[5] ZHANG, Cheng et al. Surface treatment of polyethylene terephthalate films using DBD excited by repetitive unipolar nanosecond pulses in air at atmospheric pressure. Plasma Science, IEEE Transactions on, v. 38, n. 6, p. 1517-1526, 2010.

[6] RODRÍGUEZ-MÉNDEZ, B. G. et al. PDBD with continuous liquids flows in a discharge reactor. In: Journal of Physics: Conference Series. IOP Publishing, 2015. p. 012060.

[7] KOSTOV, K. G. et al. Treatment of PET and PU polymers by atmospheric pressure plasma generated in dielectric barrier discharge in air. Surface and Coatings Technology, v. 204, n. 18-19, p. 3064-3068, 2010.

[8] DOHERTY, Kyle G. et al. Polystyrene Surface Modification for Localized Cell Culture Using a Capillary Dielectric Barrier Discharge Atmospheric-Pressure Microplasma Jet. Plasma Processes and Polymers, v. 10, n. 11, p. 978-989, 2013.

[9] LAW, Victor J.; DOWLING, Denis P. Active control metrology for preventing induced thermal damage during atmospheric pressure plasma processing of thermal sensitive materials. In: ISCS 2013: Interdisciplinary Symposium on Complex Systems. Springer Berlin Heidelberg, 2014. p. 321-331.

[10] DOHERTY, Kyle G. et al. Polystyrene Surface Modification for Localized Cell Culture Using a Capillary Dielectric Barrier Discharge Atmospheric-Pressure Microplasma Jet. Plasma Processes and Polymers, v. 10, n. 11, p. 978-989, 2013.

[11] PEKÁREK, Stanislav. Experimental study of surface dielectric barrier discharge in air and its ozone production. Journal of Physics D: Applied Physics, v. 45, n. 7, p. 075201, 2012. 
[12] KOGELSCHATZ, U.; ELIASSON, B.; EGLI, W. Dielectric-barrier discharges. Principle and applications. Journal de physique. IV, v. 7, n. 4, p. C4.47-C4.66, 1997.

[13] HEINLIN, Julia et al. Plasma medicine: possible applications in dermatology. JDDG: Journal der Deutschen Dermatologischen Gesellschaft, v. 8, n. 12, p. 968-976, 2010.

[14] CHERUTHAZHEKATT, $S$. et al. Gas plasmas and plasma modified materials in medicine. Journal of Applied Biomedicine, v. 8, n. 2, p. 55-66, 06/01/ 2010.

[15] HAIYAN, He et al. Cold deposition of large-area amorphous hydrogenated silicon films by dielectric barrier discharge chemical vapor deposition. Thin Solid Films, v. 519, n. 15, p. 5038-5042, 2011.

[16] DA PONTE, G. et al. Atmospheric pressure plasma deposition of organic films of biomedical interest. Surface and Coatings Technology, v. 205, Supplement 2, n. 0, p. S525-S528, 2011.

[17] KOSTOV, K. G. et al. Bacterial sterilization by a dielectric barrier discharge (DBD) in air. Surface and Coatings Technology, v. 204, n. 18-19, p. 2954-2959, 2010

[18] DAESCHLEIN, G. et al. Cold plasma is well-tolerated and does not disturb skin barrier or reduce skin moisture. JDDG: Journal der Deutschen Dermatologischen Gesellschaft, v. 10, n. 7, p. 509-515, 2012

[19] BRUGGEMAN, Peter et al. Optical emission spectroscopy as a diagnostic for plasmas in liquids: opportunities and pitfalls. Journal of Physics D: Applied Physics, v. 43, n. 12, p. 124005, 2010.

[20] MAHONEY, J. et al. Electrical and optical emission measurements of a capillary dielectric barrier discharge. Eur. Phys. J. D, v. 60, n. 3, p. 441-447, 2010.

[21] FANELLI, F. Optical Emission Spectroscopy of Argon-Fluorocarbon-Oxygen Fed Atmospheric Pressure Dielectric Barrier Discharges. Plasma Processes and Polymers, v. 6, n. 9, p. 547-554, 2009.

[22] MARCEL, H. H.; ET AL. Influence of the Air Humidity on the Reduction of Bacillus Spores in a Defined Environment at Atmospheric Pressure Using a Dielectric Barrier Surface Discharge. Plasma Processes and Polymers, p. NA, 2009.

[23] NIU, Z. et al. Repetitive nanosecond-pulse dielectric barrier discharge and its application on surface modification of polymers. Surface and Coatings Technology, n. 2012.

[24] SATO, T. et al. Generation and Transportation Mechanisms of Chemically Active Species by Dielectric Barrier Discharge in a Tube for Catheter Sterilization. Plasma Processes and Polymers, v. 5, n. 6, p. 606-614, 2008.

[25] SCHMIDT, M. Š. A. V. P. A. J. Optical and electrical characteristics of a single surface DBD micro-discharge produced in atmospheric-pressure nitrogen and synthetic air. Plasma Sources Science and Technology, v. 20, n. 2, p. 025009, 2011.

[26] LUO, Y.; GE, S.-R. Load Dependence of Nanohardness in Nitrogen Ion Implanted Ti6Al4V Alloy and Fractal Characterization. Journal of China University of Mining and Technology, v. 17, n. 3, p. 363-367, 9// 2007.

[27] KANG, W.-S. et al. Atmospheric-pressure cold plasma jet for medical applications. Surface and Coatings Technology, v. 205, Supplement 1, n. 0, p. S418-S421, 12/25/ 2010.

[28] QIU, X. et al. Spectroscopic study of dielectric barrier discharges in cellular polypropylene ferroelectrets. Applied Physics Letters, v. 91, n. 13, p. 132905-3, 09/24/ 2007. 